\title{
Uma meta-análise de pesquisas sobre o pensamento proporcional
}

\section{A meta-analysis of researches about proportional thinking}

\author{
Cristina Maranhão ${ }^{1}$ \\ Silvia Machado ${ }^{2}$
}

\begin{abstract}
RESUMO
O presente artigo objetiva avaliar descritores utilizados em duas pesquisas, na análise de atividades sobre o pensamento proporcional. Ambas partiram dos componentes do raciocínio proporcional divulgados por Post, Behr e Lesh, para suas análises. Por seu objetivo, emprega a metodologia designada de meta-análise qualitativa, conforme Fiorentini e Lorenzato, ao visar resultados que transcendam os daquelas pesquisas. Atingem-se tais propósitos por meio do confronto daquelas pesquisas em sua base teórica, com outros referenciais teóricos propostos por Raymond Duval, Anna Sfard, Geraldo Ávila e Elon Lages de Lima.

Palavras-chave: proporcionalidade; pensamento proporcional; metaanálise qualitativa.
\end{abstract}

\begin{abstract}
This article aims to evaluate descriptors used in two researches' analysis of activities on the proportional thinking. Both syudies were based on the proportional reasoning components proposed by Post, Behr and Lesh. According to its objective, this study employs the methodology called qualitative meta-analysis as Fiorentini and Lorenzato show, in order to find results that transcended
\end{abstract}

1 Pós-doutora em Educação Matemática pela Universidade Estadual de Campinas (FE/ UNICAMP), professora da Pontifícia Universidade Católica de São Paulo (PUC-SP), Brasil, maranhao@pucsp.br

2 Pós-doutora em Educação Matemática (IREM - Paris, França), professora da Pontifícia Universidade Católica de São Paulo (PUC-SP), Brasil, silviaam@pucsp.br 
those researches. The goals are achieves through the confrontation of those researches in their theoretical basis, with other theoretical frameworks proposed by Raymond Duval, Anna Sfard, Geraldo Ávila and Elon Lages de Lima.

Keywords: proportionality; proportional thinking; qualitative meta-analysis.

\section{Problemática}

A proporcionalidade é um tema indubitavelmente importante em Matemática e outras Ciências em âmbito escolar, e em diversas situações da atividade humana. Por isso o pensamento proporcional tem sido objeto de estudo em Educação Matemática e em uma de suas especialidades, a Psicologia da Educação Matemática, há várias décadas.

A primeira publicação conjunta dos autores Behr, Post e Lesh (1981) - no Projeto Número Racional norte-americano - divulgou a importância dos sistemas representativos na aprendizagem dos números racionais. Em outra publicação conjunta Post, Behr e Lesh (1988) relacionaram o tema da proporcionalidade às ideias algébricas em capítulo do Livro do Ano, do Conselho Nacional de Professores de Matemática norte-americano.

Por sua relevância nos Estados Unidos da América do Norte, esse livro foi traduzido ao português e veiculado no Brasil, em 1995. É trabalho que tem sido mencionado como base para pesquisas, propostas curriculares e avaliações, em âmbito nacional.

Fica explícito que os estudos realizados por esses autores os conduziram a investigações sobre proporcionalidade. Acrescentamos que em Post, Behr e Lesh (1988) eles voltam sua atenção especificamente aos componentes do raciocínio proporcional.

Em publicação que sintetiza estudos anteriores sobre o tema, pesquisadores envolvidos no Projeto Número Racional, norte-americano, consideram que há "suposições simplistas, que fundamentam a maioria dos programas de testes padronizados.” (POST; CRAMER; HAREL; KIERNEN; LESH, 1998, p. 6). Nessa síntese, clamam novas pesquisas que focalizem "ideias mais avançadas e aprofundadas" sobre o assunto, para ultrapassar reducionismos. Consideram, ainda, que, tradicionalmente, professores da escola elementar têm pouca matemática significante, mas que, no entanto, estão ensinando matemática para crianças cada e todo dia.

Com a pretensão de avaliar descritores utilizados em pesquisas que partiram dos componentes do raciocínio proporcional divulgados por Post, Behr 
e Lesh (1988; 1995), no presente artigo substituímos a designação empregada naquela publicação - raciocínio proporcional-, por pensamento proporcional, seguindo Norton (2005). Em seu artigo, o autor explica que o termo pensamento proporcional é usado para descrever os conceitos e os pensamentos requeridos para compreender: taxa de variação, proporção e proporcionalidade, incluindo escala.

Destacamos que o processo de compreensão de tais elementos conceituais se desvela por seu emprego em problemas de diversos campos matemáticos e da atividade humana, exibindo cada vez maior amplitude do conhecimento matemático operacional, até que atinja o conhecimento matemático, em seu sentido amplo, estrutural, através do trânsito adequado entre esses variados campos, conforme afirma Sfard (1987). Mas, para essa autora, tal processo não se resume a isso.

Conforme Sfard (2008), há necessidade de conciliação entre noções como aprender, conhecer, pensar, comunicar e compreender, porque são processos intrínsecos e socialmente mediados, particularmente na escola, mas não apenas nela. Cabe assim ressaltar que a noção de pensamento deve ser tomada como comunicação internalizada. Enfim, vale acrescentar que o aprofundamento devido de ideias matemáticas requer a comunicação a serviço da cognição, recaindo na metacognição: pensar sobre o que foi pensado (representado, descrito ou dito).

Sfard (2008) afirma que tais pressupostos não pretendem se contrapor aos anteriores, na psicologia da educação matemática, mas complementá-los, aprofundando a compreensão dos pesquisadores que se voltam ao ensino e aprendizagem de Matemática.

Finalmente, consideramos caber para a Educação Matemática afirmativa da mesma autora a respeito de teorias reducionistas que: "podem expressar a operacionalidade científica de seu vocabulário, mas em realidade matar seu objeto, por jogar fora algumas de suas partes vitais." (SFARD, 2008, p. 2).

Assumindo os pressupostos de Sfard, neste estudo consideramos que a formação conceitual tem a participação vital do vocabulário. Condiz com o exame de vocabulário matemático criado acerca dos tópicos matemáticos aqui focalizados, empregado antigamente e porventura obsoleto que, como tal, ao perdurar atualmente, pode atrapalhar a formação conceitual.

Isso nos conduziu a artigos da Revista do Professor de Matemática (RPM), veiculados há mais de duas décadas e voltados a professores de Matemática dos anos finais do Ensino Fundamental e do Ensino Médio. A escolha desse periódico ocorreu porque nele diversos matemáticos discutem tópicos escolares.

De fato, encontramos artigo de Ávila (1986a) em que esse matemático já discutia a apresentação do tema razões e proporções, afirmando que ela não se modernizou. 
A apresentação de tal tema se apoia ainda hoje na forma que Eudoxo o inventou para "contornar a crise dos incomensuráveis", o que lhe permitiu evitar a necessidade dos números irracionais. Ávila (1986a) completa seu argumento, considerando que a teoria das proporções de Eudoxo foi largamente empregada e aceita até o século XIX, quando foi substituída, com vantagem, pela teoria dos números reais, a qual veio legitimar a existência dos irracionais; porém os tópicos razão e proporção e regra de três continuam a ser ensinados por compilações sucessivas que perpetuam os costumes antigos.

Assim, o autor vai sugerindo ao longo do texto que se deveriam eliminar aos poucos certos termos como razão, regra de três. Enfim, ele sugere adotar o tema: variáveis proporcionais, em lugar de grandezas proporcionais, essas últimas ligadas ao problema da incomensurabilidade já superado.

O mesmo autor (ÁVILA, 1986b) volta com mais exemplos sobre o tema terminando seu artigo com a sugestão de que o nome regra de três seja abolido no ensino de matemática brasileiro.

Interessa-nos mencionar também, do mesmo periódico, os trabalhos de Lima $(1986 ; 1988)$, que discute também o assunto chamando a atenção do leitor para o fato de que "[...] antes de definir se um problema envolve proporcionalidade, e se essa quando existe é direta ou inversamente proporcional" (LIMA, 1986, p. 1), o aluno tem de perceber "a covariação" entre duas ou mais grandezas e "chegar a uma fórmula." (LIMA, 1986, p. 1).

Essa ideia requer explicações teóricas, que Duval (2002) oferece, sobre o papel da percepção distinguindo-a da visualização na aprendizagem matemática. Além disso, requer explicações sobre representações, pela complexidade da resolução de problemas envolvendo o pensamento proporcional. Pois, vimos que, muito antes, Behr, Post e Lesh (1981) já se preocupavam com o papel das representações na aprendizagem dos números racionais.

Tal complexidade nos problemas sobre proporcionalidade, considerados por Post, Behr e Lesh (1988) como bons veículos para o trabalho com diferentes formas de representação é explicada por Duval (2002) porque são eles problemas que envolvem a capacidade de fazer conversões de um tipo de registro de representação semiótica em outro. Isto não é simples, uma vez que estes são registros de representação que têm regras de funcionamento, que atendem a convenções matemáticas; daí sua complexidade.

Duval (2002) enfatiza três tipos de registros de representação semiótica: o simbólico, o gráfico e o da língua natural. O simbólico se subdivide em algébrico e numérico, podendo o numérico também ser subdividido em decimal e fracionário. A conversão de registros de representação semiótica demanda a transformação de um registro em outro, nos dois sentidos, o que aumenta a complexidade de sua aprendizagem, por requerer coordenação de registros de 
representação semiótica.

Segue um exemplo de conversão de registros de representação semiótica. $\mathrm{O}$ enunciado de um problema é apresentado no registro da língua natural:

João comprou dez pacotes de figurinhas da copa por sete reais. Seu irmão comprou vinte, seu primo quarenta, seu amigo oitenta e seu vizinho cento e sessenta pacotes desse mesmo tipo de figurinha. Quanto cada um deles pagou pelos pacotes comprados?

Tal enunciado poderia se converter em uma tabela, considerada pelo autor como registro misto: gráfico e numérico, como apoio na resolução do problema.

\begin{tabular}{|c|c|}
\hline N. ${ }^{\circ}$ de pacotes & Preço \\
\hline 10 & 7 \\
\hline 20 & \\
\hline 40 & \\
\hline 80 & \\
\hline 160 & \\
\hline
\end{tabular}

É patente que tal conversão simplifica o problema, mas segundo Duval (2002) ela não é simples para o aluno, por não ser usual no ensino, conforme suas pesquisas.

A verificação da resolução requer a volta ao enunciado do problema. Aqui vale acrescentar que ela se constitui em uma das formas de validação matemática, considerada essencial nessa área.

Tal volta ao enunciado pode se dar percorrendo os registros empregados, quando a conversão se dá em sentido inverso: do registro misto (numérico e gráfico) para o da língua natural. Mas, ela pode ocorrer através do emprego de outros registros também. Usualmente os registros empregados por alunos em uma classe são diferentes em tal processo de verificação.

Assim, há que se comunicá-los, justificando as ideias, conforme Sfard (2008).

Esse exemplo é suficiente para mostrar que a conversão de registros de representação semiótica, nos dois sentidos, é essencial na aprendizagem da Matemática, desde o início da escolaridade. Ainda, há de se considerar que ela participa do processo de conceituação, conforme Duval (2002).

Esse autor chama atenção para o seguinte: se um objeto matemático tivesse apenas uma representação, ele e ela se confundiriam. Por isso, o acesso a apenas um dos registros de representação de um objeto matemático não permite sua 
conceituação. Enfim, o autor considera que o acesso aos conceitos matemáticos depende de coordenação de ao menos dois de seus registros de representação semiótica.

Em verdade, além de um objeto matemático ter diversas representações, deve-se levar em conta que uma representação pode ser relativa a diversos objetos matemáticos. Por exemplo, o número oitenta tem diversas representações, entre elas:

$$
80=2 \times 40
$$

Aqui um objeto matemático (um número) aparece em duas representações; e a representação 2 x 40 também se refere a outro objeto matemático (a operação multiplicação).

Tal exemplo exibe tratamentos em um mesmo registro de representação, o numérico. Ao afirmar que o ensino privilegia os tratamentos, isto é, as transformações em um mesmo registro de representação semiótica, Duval (2002) enfatiza que a aprendizagem em Matemática não pode prescindir das conversões, que também participam da coordenação de registros de representação variados, de maneira essencial.

Ressaltamos que os registros de representação semiótica, por sua vez, dependem da apreensão de suas unidades representativas. Conforme Duval (2002), de um ponto de vista cognitivo, a percepção visual e, por extensão, a visualização, têm funções que são centrais nesse processo.

A percepção visual usualmente chamada de visão se refere a ter acesso direto a qualquer objeto físico: "nada é mais convincente do que o que se vê" (DUVAL, 2002, p. 320). Nesse sentido, a visão é oposta à representação, porque "a representação é algo que se toma em lugar de alguma outra coisa" (DUVAL, 2002, p. 320). Por ser a visão usualmente tomada como modelo para a noção epistemológica de intuição, essa sua função de acesso direto ao que se vê é designada como epistemológica (participa da gênese do conhecimento).

A visão também consiste em apreender simultaneamente diversos objetos ou "um campo todo" que os envolve, o que é usualmente realizado de maneira bastante imperfeita, incompleta. A visão parece dar imediatamente a completa apreensão de qualquer objeto ou situação. Essa sua função apreensiva global é designada como função sinóptica. Nesse sentido, a visão também é oposta à dedução matemática, que requer sequências de inferências justificadas, numa cadeia de afirmações matemáticas pertinentes a um dado sistema axiomático dedutivo.

Na perspectiva duvaliana da aprendizagem, realizando ambas as funções, a epistemológica e a sinóptica da visão, não se atinge o conhecimento mate- 
mático, por serem os objetos matemáticos abstratos, sendo que o acesso a eles se dá precisamente pela coordenação dos registros de representação semiótica.

Tal questionamento conduz à distinção entre visão e visualização: esta última se refere à atividade cognitiva intrinsecamente semiótica, isto é, supõe a construção de uma imagem bem distinta da percepção, por envolver organização de relações entre unidades representativas, bem como a completa apreensão de qualquer dessas organizações.

Pensamos que o exemplo dado antes da conversão de um problema envolvendo proporcionalidade em uma tabela sirva para destacar que a visão dela pode ser imperfeita. Por isso chamamos atenção à necessidade de observação, que entendemos deva ser atenta, com tempo destinado a ela. Deve ser valorizada no ensino para estabelecer relações numéricas presentes em tabela. Pois, conforme Camejo, Maranhão e Miranda (2009) sequer a compreensão de números organizados em tabelas é simples.

Aqui queremos tornar claro que tal compreensão requer organização de relações entre unidades representativas (organização de números nas células das tabelas representando o enunciado do problema), bem como a completa apreensão de qualquer dessas organizações (das relações matemáticas entre: os números na primeira coluna, os números na segunda coluna, os números nas duas colunas e os números de cada linha, para resolver o problema).

Para a análise aqui pretendida, ressaltamos a coerência entre a perspectiva de Duval (2002) e a de Sfard (1987; 2008), tanto no tocante aos aspectos operacionais e estruturais, que participam da formação conceitual matemática, como em relação aos processos intrínsecos e socialmente mediados de pensar e comunicar.

\section{Método}

Dentre os vários tipos de estudos bibliográficos ou documentais, Fiorentini e Lorenzato (2006) trazem à luz a meta-análise qualitativa. Para esses autores, essa metodologia é uma "revisão sistemática de outras pesquisas, visando realizar uma avaliação crítica das mesmas e/ou [...] produzir novos resultados ou sínteses a partir do confronto desses estudos, transcendendo aqueles anteriormente obtidos" (FIORENTINI; LORENZATO, 2006, p. 103).

No presente trabalho, selecionamos duas pesquisas do Grupo de Pesquisa em Educação Algébrica voltadas ao pensamento proporcional, a de Miranda (2009) e o trabalho de Camejo et al. (2009), especificamente com a intenção 
de produzir resultados que transcendessem os anteriores, por meio de um aprofundamento com base nos referenciais teóricos antes mencionados. Ambas contêm descritores que nos interessam. Por isso, após a breve apresentação da elaboração desses descritores naquelas pesquisas, eles são apresentados e confrontados entre si, com base nos referenciais teóricos. Desta forma, o presente estudo pode contribuir para trabalhos de grupos de pesquisa em psicologia da educação matemática.

Sobre os descritores oferecidos pelas pesquisas de referência, Miranda (2009) partiu de alguns componentes promotores do desenvolvimento do pensamento proporcional, conforme Post, Behr e Lesh (1995), com o cuidado de esclarecer e explicitar, com exemplos, o conteúdo teórico matemático subjacente. Estes foram ampliados por meio de estudo relativo à origem histórica do tema, do exame de propostas curriculares e de análise de dissertações de instituições paulistas publicadas no período 1971-2007 voltadas aos anos finais do Ensino Fundamental, cujos títulos explicitassem os termos pensamento proporcional ou proporcionalidade ou, ainda, proporções. Miranda (2009) encontrou nesse período seis dissertações de mestrado defendidas em instituições do estado de São Paulo focalizando o tema do pensamento proporcional. Dentre os documentos de orientação curricular em âmbito nacional, a autora destacou os Parâmetros Curriculares Nacionais de Matemática para o Ensino Fundamental (BRASIL, 1998) e a Proposta Curricular do Estado de São Paulo (ESTADO DE SÃO PAULO, 2008). A partir do exame documental, a autora relacionou aspectos essenciais para o desenvolvimento do pensamento proporcional, considerados descritores para a análise do conteúdo das pesquisas selecionadas. Aos quinze descritores iniciais, a partir de suas análises, a autora sentiu necessidade de acrescentar mais quatro, totalizando no final dezenove descritores, os apresentados no Quadro 1.

Com base nesses mesmos componentes, descritores e aspectos para o desenvolvimento do pensamento proporcional, Camejo et al. (2009) acrescentaram e expandiram outros, a partir de análises de produções de professores dos anos iniciais do Ensino Fundamental em problemas no assunto. Assim, constituíram quinze aspectos essenciais para a manifestação e desenvolvimento do pensamento proporcional de professores dos anos iniciais, considerados como descritores, os quais são apresentados no Quadro 2. 


\begin{tabular}{|l|l|}
\hline $\mathbf{1}$ & $\begin{array}{l}\text { Utilizar multiplicação ou divisão para resolver problemas envolvendo } \\
\text { ideias de razão ou proporção. }\end{array}$ \\
\hline $\mathbf{2}$ & $\begin{array}{l}\text { Fazer comparações numéricas ou não numéricas; trabalhar com classes de } \\
\text { equivalência de frações. }\end{array}$ \\
\hline $\mathbf{3}$ & Distiguir situações proporcionais de não proporcionais. \\
\hline $\mathbf{4}$ & Utilizar a ideia de covariação. \\
\hline $\mathbf{5}$ & $\begin{array}{l}\text { Representar situações proporcionais por meio de gráficos, tabelas, } \\
\text { símbolos, desenhos ou diagramas. }\end{array}$ \\
\hline $\mathbf{6}$ & $\begin{array}{l}\text { Relacionar proporcionalidade com ideias de medidas de comprimento, } \\
\text { superfície, volume, massa, ou capacidade etc.; efetuar conversão de } \\
\text { unidades de medida. }\end{array}$ \\
\hline $\mathbf{7}$ & Desenhar ou representar em escala. \\
\hline $\mathbf{8}$ & $\begin{array}{l}\text { Utilizar proporções na análise de dados (de uma enquete ou pesquisa) ou } \\
\text { em probabilidade. }\end{array}$ \\
\hline $\mathbf{9}$ & $\begin{array}{l}\text { Resolver problemas (tais como os que requeiram cálculos relativos a } \\
\text { impostos) envolvendo custos, taxas, porcentagem, juros, descontos. } \\
\text { Efetuar corretamente cálculos envolvendo esses tópicos. }\end{array}$ \\
\hline $\mathbf{1 0}$ & $\begin{array}{l}\text { Utilizar ideias centrais associadas aos sentidos do número racional, ou de } \\
\text { relações e operações entre eles, além de suas representações, para resolver } \\
\text { problemas envolvendo funções ou ideias associadas às funções e suas } \\
\text { representações. }\end{array}$ \\
\hline $\mathbf{1 1}$ & $\begin{array}{l}\text { Relacionar proporcionalidade com ideias de semelhança, homotetia, } \\
\text { ampliação ou redução de figuras planas. }\end{array}$ \\
\hline $\mathbf{1 2}$ & $\begin{array}{l}\text { Diferenciar grandezas diretamente proporcionais das inversamente } \\
\text { proporcionais. }\end{array}$ \\
\hline $\mathbf{1 3}$ & Resolver problemas usando a regra de três. \\
\hline $\mathbf{1 4}$ & Usar o método da falsa posição ou algo similar a ele. \\
\hline $\mathbf{1 5}$ & Usar reconfigurações geométricas precedidas de alteração de escala. \\
\hline $\mathbf{1 6}$ & $\begin{array}{l}\text { Relacionar ao menos duas das ideias centrais acerca do número racional: } \\
\text { parte-todo, razão, divisão/quociente, taxas, porcentagem, probabilidade, } \\
\text { operador, semelhança e homotetia. }\end{array}$ \\
\hline $\mathbf{1 7}$ & Utilizar a propriedade aditiva das proporções. \\
\hline $\mathbf{1 8}$ & Resolver problemas envolvendo a divisão em partes proporcionais. \\
\hline $\mathbf{1 9}$ & $\begin{array}{l}\text { Resolver problemas envolvendo inequações ou ideias associadas às } \\
\text { inequações e suas representações. }\end{array}$ \\
\hline
\end{tabular}

QUADRO 1

FONTE: (MIRANDA, 2009, p. 66-67). 


\begin{tabular}{|c|l|}
\hline a & $\begin{array}{l}\text { Utilizar estratégias pessoais para a resolução de problemas envolvendo } \\
\text { componentes do pensamento proporcional. }\end{array}$ \\
\hline $\mathbf{b}$ & $\begin{array}{l}\text { Utilizar multiplicação e divisão para resolver problemas envolvendo ideias } \\
\text { de razão e proporção. }\end{array}$ \\
\hline $\mathbf{c}$ & $\begin{array}{l}\text { Fazer comparações numéricas envolvendo os racionais e também não } \\
\text { numéricas; trabalhar com classes de equivalência de frações. }\end{array}$ \\
\hline $\mathbf{d}$ & Distiguir situações proporcionais e não proporcionais. \\
\hline e & Usar ideia de covariação. \\
\hline $\mathbf{f}$ & $\begin{array}{l}\text { Representar razões por meio de gráficos, ou tabelas, ou símbolos, ou } \\
\text { desenhos, ou, ainda, diagramas e resolver problemas envolvendo razões } \\
\text { organizadas em tabelas, gráficos etc. }\end{array}$ \\
\hline $\mathbf{g}$ & $\begin{array}{l}\text { Relacionar proporcionalidade com ideias de medidas de comprimento, ou } \\
\text { superfície, ou volume, ou massa, desenhar ou representar em escala. }\end{array}$ \\
\hline $\mathbf{h}$ & $\begin{array}{l}\text { Utilizar razões na análise de dados (de uma enquete ou pesquisa) ou em } \\
\text { probabilidade. }\end{array}$ \\
\hline $\mathbf{i}$ & $\begin{array}{l}\text { Resolver problemas envolvendo porcentagem, juros, descontos, impostos } \\
\text { e taxas. }\end{array}$ \\
\hline $\mathbf{j}$ & $\begin{array}{l}\text { Utilizar o pensamento proporcional para resolver problemas envolvendo } \\
\text { funções e suas representações ou ideias associadas a elas. }\end{array}$ \\
\hline $\mathbf{k}$ & Relacionar proporcionalidade com a ideia de semelhança. \\
\hline $\mathbf{l}$ & $\begin{array}{l}\text { Diferenciar grandezas diretamente proporcionais das inversamente } \\
\text { proporcionais. }\end{array}$ \\
\hline $\mathbf{m}$ & $\begin{array}{l}\text { Reconhecer a divisibilidade de zero e a indivisibilidade por zero ao lidar } \\
\text { com números racionais em suas diversas representações. }\end{array}$ \\
\hline $\mathbf{n}$ & $\begin{array}{l}\text { Relacionar ao menos duas das ideias acerca do número racional ou da } \\
\text { fração: parte-todo, razão, divisão/quociente, porcentagem, operador. }\end{array}$ \\
\hline $\mathbf{o}$ & $\begin{array}{l}\text { Operar com os números racionais na representação fracionária e na decimal, } \\
\text { bem como transformar uma dessas representaços na outra, imprimindo } \\
\text { significado a tais ações. }\end{array}$ \\
\hline
\end{tabular}

QUADRO 2

FONTE: (CAMEJO et al., 2009, p. 4).

Por questão metodológica, na meta-análise que segue a apresentação dos descritores, usados nas pesquisas ora analisadas, nos atemos ao confronto dos que aparecem em ambos os quadros. 


\section{A Meta-análise qualitativa dos descritores}

Apesar de considerarmos o fato de as pesquisas empregarem o tipo de nomenclatura criticado nos artigos de Ávila (1985; 1986a; 1986b), Lima (1986; 1988), ponderamos que este vocabulário deveria ser avaliado no presente estudo, principalmente para contribuir com futuras investigações que o considerem essencial ao desenvolvimento do pensamento proporcional em todos os níveis de escolaridade. Por isso a cada descritor analisado segue-se uma nova formulação dele.

Estabelecemos relações entre esses descritores e os quatro componentes do pensamento proporcional, segundo Post, Behr e Lesh (1988), por terem originado muitos trabalhos. De fato, os componentes são considerados relevantes em âmbito internacional.

Identificamos os descritores do pensamento proporcional dos itens 3 do Quadro 1 e $d$ do Quadro 2 a respeito da distinção entre situações proporcionais e não proporcionais. Pensamos que a redação do descritor do Quadro 2 seja a mais adequada:

\section{Distinguir situações proporcionais e não proporcionais}

Pode parecer que tal distinção seja de caráter perceptivo, intuitivo, como indica Lima (1986). Mas, como explica Duval (2002), deve envolver inferências e deduções; além disso, suas justificativas devem abarcar convenções matemáticas e a coordenação de registros de representação semiótica, para que se atinja o conhecimento matemático.

Conforme Sfard (1987), a resolução de problemas em diversos campos matemáticos e da atividade humana participa do processo de compreensão. É importante para que se atinja o conhecimento matemático, amplo, através do trânsito adequado entre esses variados campos. A compreensão requer, ainda, segundo Sfard (2008), a comunicação de ideias, considerando que as noções de aprender, conhecer, pensar, comunicar e compreender são intrínsecas e socialmente mediadas, particularmente na escola, mas não apenas nela, e que o aprofundamento devido de ideias matemáticas precisa atingir a metacognição. Com base nessa autora, consideramos interessante que se promova a distinção de situações proporcionais e não proporcionais, por meio da análise de enunciados de problemas de variados campos, por parte de estudantes, procurando solicitar justificativas, para atingir a metacognição através da comunicação de 
ideias matemáticas. Ponderamos finalmente que essa distinção possa preceder a tomada de decisão relativa à estratégia de resolução de problemas que requeiram outros componentes do pensamento proporcional, inspiradas em Lima (1986).

Identificamos também os descritores do pensamento proporcional dos itens 12 do Quadro 1 e $l$ do Quadro 2 relativos a diferenciar grandezas diretamente proporcionais das inversamente proporcionais.

Encontramos aí vocabulário que deveria ser alterado, pois Ávila (1986) convence ao afirmar que se deveria adotar a denominação de variáveis proporcionais ao invés de grandezas proporcionais, estas últimas ligadas a ideias geométricas e criadas para contornar o problema já superado da incomensurabilidade. Assim, segue nova formulação para esse componente do pensamento proporcional:

\section{Diferenciar variáveis diretamente proporcionais das inversamente proporcionais.}

Finalmente, retomamos Lima (1986), para quem se tem que perceber a covariação antes de se definir ser a proporcionalidade direta ou inversa para então chegar a uma fórmula. Novamente, é necessário esclarecer que pode parecer que tal definição seja de caráter perceptivo, como indica Lima (1986). Mas, pelas explicações de Duval (2002), deve envolver a visualização, que requer organização de relações entre unidades representativas, bem como a completa apreensão de qualquer dessas organizações, pois que estas participam da coordenação de registros de representação semiótica, para que o conhecimento matemático seja atingido (DUVAL, 2002). Desta forma, a visualização sobre a covariação requer que as justificativas abarquem convenções matemáticas, para a devida distinção requerida entre variáveis direta e inversamente proporcionais.

Com tantos requisitos, os problemas envolvendo a diferenciação entre variáveis proporcionais e não proporcionais requerem comunicação e mediação social nos moldes propostos por Sfard (2008). É, também, preciso que sejam propostos em variados campos, conforme Sfard (1987). Ponderamos, também, que essa diferenciação possa preceder a tomada de decisão sobre a estratégia de resolução em problemas envolvendo outros componentes do pensamento proporcional.

O descritor posto como item 1 no Quadro 1: "Utilizar multiplicação ou divisão para resolver problemas envolvendo ideias de razão ou proporção" é semelhante ao do item $b$ posto no Quadro 2: "Utilizar multiplicação e divisão para resolver problemas envolvendo ideias de razão e proporção." Embora a diferença entre um e outro pareça simples, por residir apenas na conjunção ou, 
esta pode tornar ambíguo o sentido da frase. Por isso, e com base nas explicações de Ávila (1986), segue nova formulação para ele:

\section{Usar multiplicação e divisão para resolver problemas envolvendo proporcionalidade.}

Afinal, o vocabulário participa do processo de compreensão conceitual, conforme Sfard (1987; 2008). Além disso, os trabalhos de Ávila (1986) chamam atenção ao emprego indevido do termo razão, que vem provocando inúmeras confusões na compreensão da proporcionalidade.

O descritor posto como item 2 no Quadro 1: "Fazer comparações numéricas ou não numéricas; trabalhar com classe de equivalência de frações" é semelhante ao descritor posto como item $c$ do Quadro 2: "Fazer comparações numéricas envolvendo os racionais e também não numéricas; trabalhar com classes de equivalência de frações". A segunda frase nos parece mais coerente, pois ao introduzir o termo racionais na primeira sentença, para indicar números racionais, dá sentido ao que segue sobre classes de equivalência de frações. Ponderamos, no entanto, que a igualdade de frações substitui com vantagem a equivalência, no estudo da proporcionalidade, pois esta última é tratada tanto na escola como fora dela, conforme Maranhão (2010). Além disso, segundo a autora, tal substituição pode libertar o professorado, "de fixar-se apenas no trabalho com equivalência de frações, incitando-o a investir na visualização de proporções em tabelas [...], desde os anos iniciais de escolaridade." (MARANHÃO, 2010, p. 10).

Neste artigo insistimos ser válida a libertação do trabalho com equivalência de frações, no estudo da proporcionalidade, ao nos voltarmos, em particular, aos pesquisadores em psicologia da educação matemática. Acrescentamos, porém, que no estudo da proporcionalidade, nos anos finais do Ensino Fundamental e no Ensino Médio, o trabalho com a igualdade de frações também é criticado por Ávila (1985). Este autor considera a representação da proporcionalidade pela igualdade de frações complicada e pouco prática, demonstrando que outra representação envolvendo apenas igualdade de produto de variáveis é equivalente àquela e que a precede. Por isso, neste caso sugerimos a seguinte formulação:

\section{Fazer comparações numéricas envolvendo os racionais e também não numéricas,} ao trabalhar com proporcionalidade 
O item 2 do Quadro 1 e o item $c$ do Quadro 2 se reportam ao aspecto do pensamento proporcional que envolve comparação não numérica. Post, Behr e Lesh (1988) defendem a importância das comparações não numéricas para o desenvolvimento do pensamento proporcional, sugerindo que tais comparações sejam incluídas nos currículos escolares, bem como em pesquisas sobre o tema da proporcionalidade, por dependerem, em geral, mais da experiência de vida fora da escola, do que de tópicos usualmente tratados na escola e em pesquisa.

Os itens 4, 5, 7, 10,11,12 do Quadro 1 e, respectivamente, os itens $e, f$, $g, j, k, l$ do Quadro 2 se relacionam à ideia de covariação, isto é, a variáveis interdependentes. $\mathrm{O}$ componente do pensamento proporcional correspondente a todos esses itens é, portanto, o que redigimos abaixo:

\section{Usar ideia de covariação.}

$\mathrm{Na}$ realidade, pensamos que todos esses itens sejam especificações do uso da ideia de covariação. No entanto, essas especificações não esgotam todas as possibilidades de tópicos inter-relacionados, para análises de produções de professores, ou de estudantes em pesquisas no tema e, por isso, são válidas.

Finalmente, descritores que envolvem o pensamento proporcional, em comparações envolvendo os números racionais, são contemplados nos itens 1 , 2, 6, 8, 9, 16 do Quadro 1, que correspondem, respectivamente, aos itens $b, c, g$, $h, i, n$ do Quadro 2. Relacionados a tópicos prioritariamente escolares, visando ao desenvolvimento da ideia de número racional, captam várias facetas dessa ideia, mas nem todas.

Por isso, finalmente, é preciso destacar que outros descritores podem ser criados, dependendo naturalmente do nível de ensino, a que se voltem as pesquisas que os empreguem.

Assim, esperamos que o presente estudo possa trazer contribuições ao desenvolvimento de futuras investigações no tema, com implicações para o ensino.

\section{REFERÊNCIAS}

ÁVILA, G. Eudoxo, Dedekind, números reais e ensino de Matemática. Revista do Professor de Matemática (RPM), Rio de Janeiro, n. 7, p. 1-8, 1985. 
. Razões, proporções e regra de três. Revista do Professor de Matemática (RPM), Rio de Janeiro, n. 8, p. 1-8, 1986a.

. Ainda sobre a regra de três. Revista do Professor de Matemática (RPM), Rio de Janeiro, n. 9, p. 1-9, 1986b.

BEHR, M.; POST, T.; LESH, R. Construct analyses, manipulative aids, representational systems and the learning of rational numbers. In: TALL, D. O.; VINNER, S. (Orgs.). Proceedings of the 21th Conference of the International Group for Psychology of Mathematics Education. Grenoble: PME, 1981, p. 203-209. v. 5.

BRASIL. Secretaria de Educação Fundamental. Parâmetros curriculares nacionais: Matemática. Brasília: MEC/SEF, 1998.

CAMEJO, A.; MARANHÃO, C.; MIRANDA, M. R. Ideias de professoras dos anos iniciais sobre números racionais. In: IV SEMINÁRIO INTERNACIONAL DE PESQUISA EM EDUCAÇÃO MATEMÁTICA/SIPEM. Sociedade Brasileira de Educação Matemática. Anais... Brasília-DF: SBEM, UCB, 2009, p. 1-11. CD-ROM.

DUVAL, R. Representation, vision and visualization: cognitive functions in mathematical thinking: Basic issues for learning. In: HITT, F. (Ed.). Representations and mathematics visualization. México, D.F.: Departamento de Matemática Educativa del Cinestav - IPN, 2002. p. 312-335.

ESTADO DE SÃO PAULO. Secretaria Estadual de Educação Fundamental. Proposta curricular do Estado de São Paulo. São Paulo: SEE-SP, 2008.

FIORENTINI, D.; LORENZATO, S. Investigação em educação matemática: percursos teóricos e metodológicos. Campinas: Autores Associados, 2006.

LIMA, E. L. Que são grandezas proporcionais? Revista do Professor de Matemática(RPM), Rio de Janeiro, n. 9, p. 21-29, 1986.

. Novamente a proporcionalidade. Revista do Professor de Matemática (RPM), Rio de Janeiro, n. 12, p. 8-12, 1988.

MARANHÃO, C. Uma meta-análise de pesquisas sobre o pensamento proporcional. In: X ENCONTRO NACIONAL DE EDUCAÇÃO MATEMÁTICA. Educação matemática, cultura, diversidade. Anais... Salvador: SBEM, 2010. v. 1. p. 1-11. CDROM.

MIRANDA, M. R. Pensamento proporcional: uma metanálise qualitativa de dissertações. Dissertação (Mestrado) - Faculdade de Ciências Exatas e Tecnologia, Pontifícia Universidade Católica de São Paulo, São Paulo, 2009.

NORTON, S. J. The construction of proportional reasoning. In: CHICK, H. L.; 
VINCENT, J. L. (Eds.). Proceedings of $29^{\text {th }}$ Conference of the International Group for the Psychology of Mathematics Education. Melbourne: PME, 2005. v. 4. p. 17-24.

POST, T.; BEHR, M.; LESH, R. Proportionality and the development of prealgebra understandings. In: COXFORD, A.; SHULTE, A. (Eds.). The ideas of algebra, K-12: 1988 Yearbook of the National Council of Teachers of Mathematics. Reston, VA: The Council, 1988. p. 78-90.

A proporcionalidade e o desenvolvimento de noções pré-álgebra. In: COXFORD, A. F.; SHUlTE, A. P. (Orgs.). As idéias da álgebra. Tradução de: DOMINGUES, H. H. São Paulo: Atual, 1995. p. 89-103.

POST, T.; CRAMER, K.; HAREL, G., KIERNEN, T.; LESH, R. Research on rational number, ratio and proportionality. IN: NATIONAL COUNCIL OF TEACHERS OF MATHEMATICS (Orgs.). Proceedings of the $20^{\text {th }}$ Annual Meeting of the North American Chapter of the International Group for the Psychology of Mathematics Education. Raleigh, NC: PME, 1998. v. 1. p. 89-93.

SFARD, A. Two conceptions of mathematical notions: operational and structural. In: BERGERON, J. C.; HERSCOVICZ, N.; KIERAN, C. (Orgs.). Proceedings of the Eleventh International Conference of the International Group for the Psychology of Mathematics Education. Montréal: Université de Montréal, 1987. v. III. p. 162-169.

. Thinking as communicating: human development, the growth of discourses, and mathematizing. Cambridge, UK: Cambridge University Press, 2008. 